\title{
Langues sub-étatiques et principes du droit : la dialectique de l'épreuve réciproque
}

\section{Eddy Pognon et Jean-Michel Ragald}

\section{(2) OpenEdition}

\section{Journals}

Édition électronique

URL : http://journals.openedition.org/plc/294

DOI : $10.4000 /$ plc. 294

ISSN : 2117-5209

Éditeur

L'Harmattan

Édition imprimée

Date de publication : 1 janvier 2002

Pagination : 137-176

ISSN : 1279-8657

\section{Référence électronique}

Eddy Pognon et Jean-Michel Ragald, «Langues sub-étatiques et principes du droit : la dialectique de

l'épreuve réciproque », Pouvoirs dans la Caraïbe [En ligne], 13 | 2002, mis en ligne le 02 mai 2011, consulté le 19 avril 2019. URL : http://journals.openedition.org/plc/294 ; DOI : 10.4000/plc.294 


\title{
Langues sub-étatiques et principes du droit : la dialectique de l'épreuve réciproque
}

\author{
Par Eddy POGNON \\ Diplômé en droit public \\ Directeur de cabinet du Président du \\ Conseil général de Guyane \\ \& Jean-Michel RAGALD \\ Diplômé en droit communautaire \\ Collaborateur de Député
}

La pluralité de l'expression idiomatique intra-nationale a été appréhendée diversement par les droits nationaux et international. Cette circonstance a partiellement expliqué le caractère tardif de la définition des langues infra-étatiques. Selon la Charte européenne sur les langues régionales ou minoritaires (ci-après la Charte) du 5 novembre 1992, « pratiquées traditionnellement sur un territoire d'un Etat»par ses ressortissants constituant «un groupe numériquement inférieur au reste de la population de l'Etat », elles sont « différentes de la (des) langue(s) officielle(s) de cet Etat ${ }^{1} »$. La pratique traditionnelle, l'emploi par les nationaux, le nombre inférieur de locuteurs eu égard au reste de la population de l'Etat et la non-officialité d'Etat en sont les composantes.

Le premier critère de la définition se dédouble. D'une part, il repose sur une assise historique ${ }^{2}$ et une transmissibilité inter-

\footnotetext{
${ }^{1}$ Art. $1^{\text {er }}$ de la Charte européenne des langues régionales ou minoritaires. Donc, en principe, le suédois, langue officielle de la Finlande, n’y serait pas langue régionale ou minoritaire au sens de la Charte. Pourtant, le Comité des experts a adopté une jurisprudence opposée à son sujet.

2 Rapport explicatif de la Charte européenne des langues régionales ou minoritaires, http://conventions.coe.int/treaty/fr/Reports/Html/148.htm, §33, p. 5 .
}

$\operatorname{PDLC~n}^{\circ}$ 13, 2002 
générationnelle. L'approche historique souligne une variété d'origines externes de langues sub-étatiques et une «évolutivité » linguistique. D'autre part, il implique la dénotation d'une zone d'influence ${ }^{3}$ reposant sur une « assise territoriale traditionnelle ${ }^{4}$ » d'où le renvoi de la Charte $\mathrm{au}$ « territoire sur lequel ces langues sont pratiquées ${ }^{5} »$. La notion de langue régionale suppose «la définition d'un champ géographique d'application autre que celui de l'Etat dans son entier ${ }^{6} »$. La Convention-cadre pour la protection des minorités nationales (ci-après la Convention-cadre) se réfère aux «aires géographiques d'implantation substantielle ou traditionnelle des personnes appartenant à des minorités nationales ${ }^{7} »$. Selon le Conseil de l'Europe, l'adjectif «traditionnelle» ne renverrait pas aux minorités historiques, «mais à celles qui vivent toujours sur la même aire géographique ${ }^{8}$ » dont l'existence est une condition de l'usage d'une langue minoritaire dans les rapports entre les personnes appartenant à des minorités nationales et des autorités administratives ${ }^{9}$. Le principe de territorialité admis par la Cour européenne des droits de l'homme ${ }^{10}$ qui, au-delà de la délimitation de la zone d'influence, justifie les interventions d'institutions sub-étatiques, n'est nullement remis en cause par ces traités internationaux.

\footnotetext{
${ }^{3}$ L. n ${ }^{\circ}$ 1-46 du 11 janvier 1951 relative à l'enseignement des langues et dialectes locaux ; JORF, 13 janvier 1951, p. 483.

${ }^{4}$ http://conventions.coe.int/treaty/fr/Reports/Html/148.htm, § 33, p. 5.

${ }_{6}^{5}$ Art. $8, \S 1$ de la Charte européenne des langues régionales ou minoritaires.

${ }^{6}$ http://conventions.coe.int/treaty/fr/Reports/Html/148.htm, § 33, p. 5.

${ }^{7}$ Art. $10 \S 2$ et $14 \S 2$ de la Convention-cadre pour la protection des minorités nationales.

${ }^{8}$ Conseil de l'Europe, Rapport explicatif de la Convention-cadre pour la protection des minorités nationales,

http://conventions.coe.int/treaty/fr/Reports/Html/157.htm, p. 10

${ }^{9}$ Art. $10 \$ 2$ de la Convention-cadre sur la protection des minorités nationales. ${ }^{10} \mathrm{CEDH}, 23$ juillet 1968, relative à certains aspects du régime linguistique en matière d'enseignement en Belgique, A6, Req. $\mathrm{n}^{\circ}$ 00001474/62, 00001677/62, 00001691/62, 00001769/63, 00001994/63, 00002126/64, Rec. II, p. 87 CEDH, 2 mars 1987, A113, Req. $n^{\circ}$ 00009267/81, Mathieu Mohin et Clerfayt c./Belgique, point $\mathrm{n}^{\circ} 57$.
}

\section{$\operatorname{PDLC} n^{\circ}$ 13, 2002}

口 
Cependant, des langues non rattachées à une aire géographique particulière peuvent remplir les critères mentionnés à l'article $1^{\text {er }}$ de la Charte $^{11}$ sans besoin de faire coïncider un territoire d'une langue subétatique avec une circonscription administrative du fait de la complexité de la répartition géographique des locuteurs ${ }^{12}$. Il s'agit de tenir compte de la situation des minorités historiques. Instaurer ou encourager un enseignement dans ou d'une langue sub-nationale dans les territoires autres que ceux d'influence ${ }^{13}$ assure la mobilité et la valorisation des langues dépourvues de territoire.

Le second critère a pour fonction d'évincer les langues parlées uniquement par des étrangers. Une «minorité nationale » se compose de citoyens d'un Etat entretenant avec eux d'étroits liens tout en présentant des «caractéristiques linguistiques spécifiques ${ }^{14} »$. La région est un territoire sub-étatique établi sur des caractères communs dont, le cas échéant, la langue.

Le troisième critère dépeint une situation démographique exclusive de la langue majoritaire ${ }^{15}$.

Le dernier fait de la langue régionale ou minoritaire une langue non officielle, officielle sub-étatique ou d'autres Etats ${ }^{16}$.

L'insertion des droits linguistiques dans les droits de l'homme ${ }^{17}$, au-delà des minorités, suggère une réflexion approfondie de l'application des principes juridiques. Une conception classique de leur

${ }^{11}$ Art. $1^{\text {er }}$ littera $\mathrm{b}$ de la Charte européenne des langues régionales ou minoritaires.

${ }^{12}$ http://conventions.coe.int/treaty/fr/Reports/Html/148.htm, § 59, p. 8.

${ }_{13}^{13}$ Art. $8 \S 2$ de la Charte européenne des langues régionales ou minoritaires.

${ }^{14}$ Art. $1^{\text {er }}$ de la proposition de protocole additionnel à la Convention de sauvegarde des droits et libertés fondamentales concernant les personnes appartenant à des minorités nationales,

http://stars.coe.fr/ta/ta93/frec1201.htm, p. 3

${ }^{15}$ http://conventions.coe.int/treaty/fr/Reports/Html/148.htm, p. 1.

${ }^{16}$ A l'inverse de l'opinion du professeur Guy Carcassonne, l'arabe, langue officielle d'autres pays, peut être une langue minoritaire en France.

${ }^{17} \mathrm{http} / / /$ perso.wanadoo.fr/Idh/publications/dossdocs/charte_ipw.htm.

$\operatorname{PDLC} n^{\circ} 13,2002$ 
mise en œuvre empêche, dans certains Etats, l'érection des droits linguistiques subjectifs et/ou collectifs. On comprend que, face à la souveraineté des Etats, les actes internationaux d'incitation aient été d'abord sporadiques ${ }^{18}$.

La malléabilité du droit linguistique international et l'étude comparative de certains droits nationaux entraînent différentes formes de régulation juridique. Conceptions des «libertés » et «droit»sont souvent opposés. La capacité de ces «droits» à autoriser des applications différenciées de principes du droit conduit à dépasser les considérations doctrinales se cantonnant à l'antagonisme entre la mise en œuvre desdits principes et la consécration de «droits subjectifs » ou « collectifs » inscrits dans le droit à la langue. En somme, tout «droit de la langue » ne formule pas un «droit à la langue ». Seuls quelques exemples étrangers témoignent d'une conciliation entre principes fondamentaux attachés à la personne et des droits subjectifs et collectifs d'usage d'une langue sub-étatique.

De ce fait, l'application de ces principes en tant que garantie de l'expression du pluralisme idiomatique peut répondre de prime abord à une conception classique (I). Mais, ce pluralisme s'exprimera mieux au travers de la recherche d'un statut effectif en faveur des langues infranationales, tendance moderne qui met à l'épreuve des principes qui, déjà autorégulateurs, font face à des contraintes politiques et juridiques complémentaires ou contradictoires (II).

${ }^{18}$ Traité de Versailles sur la reconnaissance de l'indépendance de la Pologne et la reconnaissance des minorités du 28 juin 1919 ; Traité de Saint-Germainen-Laye sur la reconnaissance de l'indépendance de la Tchécoslovaquie et la protection des minorités du 10 septembre 1919 (WACHSMANN P., «Les droits de l'homme », Connaissance du droit, Ed. 1992, pp. 7-8).

\section{$\operatorname{PDLC~} n^{\circ}$ 13, 2002}




\section{I. - LES PRINCIPES DU DROIT : UNE GARANTIE DU} PLURALISME DE L'EXPRESSION IDIOMATIQUE

Les principes de liberté (A) et d'égalité (B) sont utiles à l'encouragement de la pluralité idiomatique et indissociables d'un traitement minimal correct des langues concernées.

\section{A. - L'approche minimaliste des libertés linguistiques}

Nombreux sont les Etats, dont la France, à pratiquer une "conception classique des libertés" en matière linguistique (1). Les conséquences juridiques ont ainsi pu être relevées (2).

\section{L'application de la "conception classique des libertés"}

L'utilisation d'une langue relève de la liberté individuelle dont les formes sont multiples. La rédaction d'actes juridiques interpersonnels dans une langue sub-nationale ressortit de la liberté contractuelle et ne saurait constituer un motif d'invalidité ${ }^{19}$. Cette validité produit un effet relatif ${ }^{20}$. Son opposabilité aux tiers intéressés ${ }^{21}$ dépend de la maîtrise de cette langue par ceux-ci ou, à défaut, de leur information sur le contenu de l'acte par celui qui le fait prévaloir ${ }^{22}$.

La liberté de choix de la langue exprime a priori une liberté de communication des pensées et des opinions. Toute personne peut "parler, écrire, imprimer librement, sauf à répondre de l'abus de cette liberté dans les cas déterminés par la loi ${ }^{23}$ ".

19 Art. 9 \& 2 lettre a) de la Charte européenne des langues régionales ou minoritaires.

${ }^{20}$ Idem, art. $9 \S 2$ lettre c) ; Idem, art. 9 § 2 lettre b)

${ }^{21}$ Idem, art. 9 \$ 2 lettre b).

${ }^{22}$ Idem, art. $9 \S 2$ lettre b)

${ }^{23}$ Art. 11 de la Déclaration des droits de l'homme et du citoyen de 1789.

$\operatorname{PDLC~n}^{\circ}$ 13, 2002 
C'est pourquoi la mise en œuvre de l'article 2 de la Consitution selon laquelle le français est la langue de la République ne saurait méconnaître l'importance de la liberté d'expression et de communication en matière d'enseignement, de recherche et de communication audiovisuelle ${ }^{24}$. Il incombe au législateur de garantir une liberté constitutionnellement admise ${ }^{25}$.

Aussi, il connaît du cadre d'expression des langues infraétatiques. La loi Deixonne du 11 janvier 1951 a défini les modalités de l'organisation de l'enseignement des langues et dialectes locaux dont le caractère facultatif s'inspire a priori de la liberté d'option. Il n'y a pas d'obligation de recevoir l'enseignement de ces idiomes.

Cette liberté de choix est néanmoins écartée en maternelle et dans le primaire car la liberté d'appréciation de l'instituteur prévaut. En vertu d'instructions pédagogiques adressées aux recteurs, il apprécie l'opportunité d'en faire appel, singulièrement lorsqu'il y a profit pour en enseignement, notamment du français ${ }^{26}$. L'étude des notions élémentaires de lecture et d'écriture des langues régionales et celle des morceaux choisis de la littérature sont assujetties à sa volonté et à sa demande ${ }^{27}$. La Charte, de son côté, se distingue de la loi Deixonne tant du point de vue de l'intensité du recours aux langues sub-nationales que du respect de l'autonomie de volonté. Elle fait dériver de la volonté des familles des élèves l'éducation préscolaire ${ }^{28}$ ou une partie substantielle de celle-ci ${ }^{29}$, l'enseignement primaire ${ }^{30}$ ou une partie substantielle de celui-ci ${ }^{31}$ dans ces langues ${ }^{32}$.

${ }^{24}$ Déc. $n^{\circ}$ 99-412 DC du 15 juin 1999 ; JORF, 18 juin 1999, p. 8964

25 Concernant l'article 11 de la DDHC, Cons. Const. 94-345 DC, 29 juillet 1994 , cons. ${ }^{\circ} 5$.

${ }^{26}$ Art. 2 de la loi $\mathrm{n}^{\circ} 51-46$ du 11 janvier 1951 relative à l'enseignement des langues et dialectes locaux, préc., supra note $\mathrm{n}^{\circ} 3$.

${ }_{27}^{27}$ Idem, art. 3.

${ }^{28}$ Art. 8 § 1 lettre a), i de la Charte européenne des langues régionales ou minoritaires.

${ }^{29}$ Idem, art. 8 \& 1 lettre a), ii.

${ }^{30}$ Idem, art. $8 \$ 1$ lettre b), i.

${ }^{31}$ Idem, art. 8 § 1 lettre b), ii.

\section{$\operatorname{PDLC~} n^{\circ}$ 13, 2002}


En revanche, dans le secondaire, l'option "langue et culture régionales" de trois heures par semaine, l'enseignement bilingue avec composition éventuelle en langue régionale aux épreuves d'histoiregéographie du brevet au collège et l'option facultative de langues régionales au lycée assurent la liberté de choix des élèves, également garantie par la Charte qui renvoie aussi à la volonté de la famille, "le cas échéant ${ }^{33}$ ",

Les cours et stages dans les écoles normales durant la formation professionnelle portant sur la langue, le folklore, la littérature et les arts populaires locaux destinés aux élèves-maîtres et élèves-maîtresses voués à enseigner dans une région à spécificité linguistique sont facultatifs ${ }^{34}$.

La loi Deixonne, applicable aux langues transfrontalières et insulaires, avait initialement une portée réduite aux zones d'influence bretonne, basque, catalane et occitane avant qu'elle ne fut étendue par des décrets ultérieurs au corse ${ }^{35}$, au tahitien ${ }^{36}$ et aux langues mélanésiennes $^{37}$, puis par la loi d'orientation pour l'outre-mer aux "langues régionales en usage dans les départements d'outre-mer ${ }^{38}$ ". La formation assurée dans les établissements scolaires et d'enseignement

${ }^{32}$ Idem, art. $8 \S 1$ lettre a), iii et art. $8 \S 1$ lettre b), iv.

${ }^{33}$ Idem, art. 8 \& 1 lettre c), iv

${ }^{34}$ Art. 5 de la loi $n^{\circ}$ 51-46 du 11 janvier 1951 relative à l'enseignement des langues et dialectes locaux, préc., supra note 3 .

${ }^{35}$ Décret $\mathrm{n}^{\circ} 74-334$ du 16 janvier $1974 ; J O R F, 18$ janvier 1974, p. 694

${ }^{36}$ Décret n $^{\circ} 81-553$ du 12 mai $1981 ; J O R F, 16$ mai 1981, p. 1489.

${ }^{37}$ Décret $\mathrm{n}^{\circ} 92-1162$ du 20 octobre 1992 ; JORF, 23 octobre 1992, p. 74.767 (ajië, drehu, nengone, paicï).

38 Art. 34 de la loi $\mathrm{n}^{\circ}$ 2000-1207 du 13 décembre 2000 d'orientation pour l'outre-mer; JORF, 14 décembre 2000, p. 19.760, avec pour conséquences l'insertion d'une option «langue et culture créoles» dans le concours professoral, la formation des enseignants en la matière, le renforcement des enseignements optionnels dans le secondaire et la création de CAPES y afférents (BO du 11 mars 2001 relatif au décret instituant le CAPES de créole; BO du 7 octobre 2001 définissant les programmes et les modalités d'organisation des épreuves).

$\operatorname{PDLC~n}{ }^{\circ} 13,2002$ 
supérieur pouvait déjà comporter "un enseignement, à tous les niveaux, de langues et cultures régionales ${ }^{39}$ ". Les lois portant statut de certains territoires valorisent l'enseignement des langues de culture propre ${ }^{40}$.

Le législateur ne s'est pas cantonné à l'enseignement. Dans de nombreux secteurs dont le commerce, un "usage obligatoire mais non exclusif de la langue française" est prévu", sous réserve des dispositions sur l'usage des langues régionales de France ${ }^{42}$. Le libre recours aux langues régionales procède de la non-exclusivité.

\section{Les conséquences juridiques de la "conception classique des} libertés"

L'utilisation des langues sub-étatiques intègre les droits civils et politiques" 43 La "conception classique des libertés" est citée "par opposition aux droits". En attestent les positions de la Belgique et de la Commission européenne des droits de l'homme au sujet du Protocole $n^{\circ} 2$ et des articles $8^{44}$ et $14^{45}$ de la Convention européenne

\footnotetext{
${ }^{39}$ Art. $1^{\text {er }}$ de la loi $\mathrm{n}^{\circ} 89-484$ du 10 juillet 1989 d'orientation sur l'éducation ; JORF, 14 juillet 1989, p. 8.860

${ }^{40}$ Pour les langues polynésiennes, art. 115 de la loi n ${ }^{\circ}$ 96-312 du 12 avril 1996 organique relative à la Polynésie, JORF, 13 avril 1996, p. 5.695 ; pour les langues «kanak», art. 215 de la loi n ${ }^{\circ}$ 99-209 du 19 mars 1999 organique relative à la Nouvelle-Calédonie ; JORF, 21 mars 1999, p. 4197 ; pour les langues mahoraises, art. 23 de la loi $\mathrm{n}^{\circ} 2001-616$ du 11 juillet 2001 relative à Mayotte, JORF, 13 juillet 2001, p. 11199.

${ }^{41}$ Circulaire du 20 mars 1996 concernant l'application de la loi n 94-665 relative à l'emploi de la langue française.

${ }_{42}$ Art. 21 de la L. n ${ }^{\circ}$ 4-665 du 4 août 1994 ; JORF, 5 août 1994, p. 11.392.

43 Art. 27 du Pacte international relatif aux droits civils et politiques des Nations-Unies.

${ }^{44} 1$. «Toute personne a droit au respect de sa vie privée et familiale, de son domicile et de sa correspondance. » 2 . «Il ne peut y avoir d'ingérence d'une autorité publique dans l'exercice de ce droit que pour autant que cette ingérence est prévue par la loi et qu'elle constitue une mesure qui, dans une société démocratique, est nécessaire à la sécurité nationale, à la sûreté publique, au bien-être économique du pays, à la défense de l'ordre et de la santé ou de la morale, ou à la protection des droits et libertés d'autrui ».
}

\section{$\operatorname{PDLC~} n^{\circ}$ 13, 2002}


des droits de l'homme (ci-après CEDH) énoncés lors de l'examen du régime linguistique belge en matière d'enseignement.

Le status libertatis suppose de "simples devoirs d'abstention" issus "d'interdictions d'interdire". En l'absence d'obligation positive émanant du Protocole P1-2, s'impose aux Etats parties la proscription d'entraver l'instruction de personnes soumises à leur juridiction. Néanmoins, l'article 2 du Protocole additionnel P1-2 consacre un droit égal à l'instruction, impliquant, selon la Cour européenne des droits de l'homme, une "garantie collective". Combiné avec l'article $14 \mathrm{CEDH}$ il n'assure pas le "droit" des enfants ou parents à une instruction dispensée dans la langue de leur choix. La non-discrimination en raison de la langue n'exige pas forcément la consécration de "droits spécifiques". La liberté est exclusive du "droit à la langue subétatique".

Si le droit international cherche à favoriser la reconnaissance d'un droit à l'emploi des langues propres à certaines minorités ${ }^{46}$, l'objectif n'est pas à tout prix et en tout acte de leur réserver un statut particulier mais plutôt d'empêcher des restrictions étatiques à l'exercice de libertés. Cette lecture minimaliste du droit international n'a pas convaincu le Gouvernement français en 1981 de la constitutionnalité de l'article 27 du Pacte international des droits civils et politiques des Nations-Unies du 16 décembre 1966 en vertu duquel "dans les Etats où il existe des minorités ethniques, [...]linguistiques, les personnes appartenant à ces minorités ne peuvent être privées du droit $[. .$.$] en commun avec les autres membres de leurs groupes [...]$ d'employer leur propre langue".

La suspicion à l'égard du mot "droit" devait circoncrire l'engagement de la France à la non-entrave de la pratique d'une langue au titre des libertés.

45 «La jouissance des droits et libertés reconnus dans la [...] Convention doit être assurée, sans distinction aucune, fondée notamment sur [...] la langue, [...] l'appartenance à une minorité ».

${ }_{46}$ Art. 27 du Pacte international relatif aux droits civils et politiques des Nations-Unies signé à New York le 16 décembre 1966.

$\operatorname{PDLC} n^{\circ}$ 13, 2002 
Devant ce précédent et aux fins de l'examen de la compatibilité de la Charte avec l'article $1^{\text {er }}$ de la Constitution française, le professeur Guy Carcassonne préconisa une déclaration interprétative du concept de "groupe" tiré de la Charte. "Addition d'individus", il n'est pas "une entité distincte de ceux qui la composent, pouvant avoir une personnalité propre et jouir de droits dont elle serait titulaire".

Selon l'une des réserves françaises, l'application de la Charte en France ne vise ni la reconnaissance, ni la protection des minorités, mais la promotion du patrimoine linguistique européen. Les "droits colectifs" des locuteurs des langues infra-étatiques sont exclus de la notion de "groupe".

Dans ces conditions, la difficulté de contourner le piège constitutionnel tenait dans l'opposition entre une "conception classique des libertés" et la reconnaissance de "droits" spécifiques. Le Conseil d'Etat l'avait perçu dès le 24 septembre 1996, en constatant que "les obligations prévues aux articles 9 et 10 prévoient un véritable droit à l'utilisation de langues régionales ou minoritaires dans les rapports avec la justice et les autorités administratives. Or, les prescriptions de l'article 9 rendant impossible l'usage d'une langue autre que le français devant les tribunaux pénaux, civils et administratifs ne pourraient être appliquées sans que soient méconnues les obligations résultant de l'article 2 de la Constitution. [...] l'Etat ne saurait raisonnablement esquiver la difficulté créée par les prescriptions de l'article 10 relatif à l'usage des langues régionales ou minoritaires par les autorités administratives et les services publics, en retenant dans cet article quelques mesures marginales, apparemment compatibles avec l'obligation d'utiliser le français à condition que ce ne soit pas à titre exclusif. Cette option ne permettrait pas de donner consistance à la politique qu'il se serait engagé à mettre en œuvre à la partie II, et qui consiste bien à promouvoir l'usage de ces langues dans la vie publique au même titre que dans la vie privée".

Certes, la thèse de l'inconstitutionnalité de cette Charte n'a pas été partagée par tous. Sur la base de son analyse téléologique amenant sur le terrain de la sauvegarde de ces langues, non sur celui de la concurrence avec des langues officielles d'Etat, le professeur Guy Carcassonne proposa une application minimale du texte. La

PDLC n ${ }^{\circ}$ 13, 2002 
France y a souscrit, particulièrement au sujet de l'enseignement préscolaire à secondaire, de la publication des actes des collectivités territoriales et des entreprises, de la rédaction des consignes de sécurité, de la promotion des contacts entre locuteurs de la même langue habitant dans des Etats différents, de la traduction des lois les plus importantes, de la toponymie, des médias et de la traduction d'œuvres produites dans d'autres langues ${ }^{47}$.

Mais saisi au titre de l'article 54 de la Constitution, le Conseil constitutionnel rejeta toute reconnaissance de "droits" particuliers. Par sa décision du 15 juin 1999, devant la seule "force normative" herméneutique de la déclaration "en cas de litige", il a procédé "au contrôle de constitutionnalité des engagements souscrits par la France indépendamment de celle-ci”. Les principes fondamentaux de la République comme l'unicité du peuple français empêchent la consécration de "droits collectifs" à quelque groupe que ce soit, défini par son origine, sa culture ou sa croyance.

\section{B. - Le principe d'égalité au regard des relations inter- linguistiques}

La neutralité commande les relations inter-linguistiques verticales. La protection et l'encouragement des langues sub-étatiques ne sauraient se faire au détriment des langues officielles et de la nécessité de les apprendre. Il s'agit de ne pas désavouer les langues d'Etat. Mais, il existe aussi des langues officielles infra-étatiques. Les différences de traitement juridique intra-étatique donnent à réfléchir sur les discriminations "verticales" (1) ou "horizontales" (2).

\section{Les difficultés d'admission de l'existence de "discriminations} verticales"

La diversité des régimes juridiques des langues sub-étatiques est observable par la comparaison des droits internes et par l'appréciation

${ }^{47}$ Le Monde hebdomadaire, 26 juin 1999, p. 10.

PDLC n ${ }^{\circ}$ 13, 2002 
des discriminations intra-étatiques. Les langues sub-étatiques ont rarement bénéficié d'une consécration juridique entière caractérisée par une officialisation des langues infra-étatiques sans discrimination.

L'absence de leur promotion résulte de leur non-prise en compte ou, plus rarement, de leur inexistence dans un pays. Dans ce dernier cas, la question de la "discrimination verticale" ne se pose pas ${ }^{48}$. Elle est inopportune dans de rares hypothèses où il n'y a aucune langue commune à tous les ressortissants d'un Etat.

Généralement, les langues officielles d'Etat ont été valorisées aux niveaux constitutionnel, législatif et réglementaire. En cas de négation de réalités linguistiques sub-étatiques, la politique d'assimilation linguistique à travers une unité, forcée ou non, autour de la langue officielle d'Etat caractérise-t-elle une forme de "discrimination verticale" ?

La controverse et les subtilités sont trop étendues pour adopter une position linéaire. L'existence d'une « discrimination verticale » est tacitement réfutée par le Conseil de l'Europe dans son rapport explicatif de la Charte: «Les mesures définies par chaque Etat en faveur de l'utilisation d'une langue nationale ou officielle ne constituent pas une discrimination à l'égard des langues régionales pour seul motif que les mêmes mesures ne sont pas prises au profit de ces dernières ${ }^{49} \gg$.

De plus, le droit linguistique est avant tout un droit d'Etat et le souci européen est d'éluder les antagonismes entre les langues d'Etat et infra-nationales ${ }^{50}$.

Le refus d'admettre la notion de «discrimination verticale» peut s'accompagner de la recherche d'une complémentarité interlinguistique verticale. Ainsi, la rupture constitutionnelle avec une dynamique dépréciative des langues régionales a servi à relever

${ }^{48}$ Pour le cas du Liechtenstein, rapport du Comité des experts de la Charte du 9 février 2001, ECRLM (2001) 5.

${ }^{49} \mathrm{http}: / /$ conventions.coe.int/treaty/fr/Reports/Html/148.htm, § 72, p. 10.

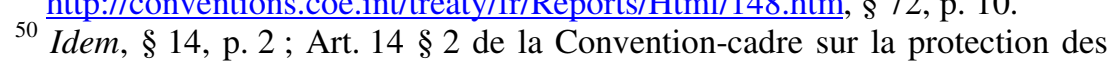
minorités nationales.

\section{$\operatorname{PDLC~n}{ }^{\circ} 13,2002$}


l'éclectisme de la langue française qui «évolue, comme toute langue vivante, en intégrant dans son vocabulaire usuel des termes de diverses sources, qu'il s'agisse d'expressions issues de langues régionales, de vocables dits populaires ou de mots étrangers ${ }^{51} \gg$.

Certes, dès l'époque révolutionnaire, l'absence d'égalité découlait du souci d'assurer une hégémonie du français ${ }^{52}$. L'article 2 de la Constitution française ne fonde pas l'unicité linguistique au titre du principe de liberté d'expression, mais seulement une unité linguistique malgré la diversité. Lorsqu'il s'agit de l'interpréter, les proscriptions sectorielles de l'emploi des langues territoriales ont pu être mieux appréhendées. L'article 2 de la Constitution française impose «l'usage du français aux personnes morales de droit public et aux personnes privées dans l'exercice d'une mission de service public, ainsi qu'aux usagers dans leurs relations avec les administrations et services publics ${ }^{53} \gg$.

La faculté de recours à une langue régionale dans l'administration est ainsi limitée. «Ne pas prononcer la censure, mais simplement rappeler, par la réserve d'interprétation, que l'usage du français s'impose, suffisait à priver quiconque eût prétendu s'en prévaloir d'un droit acquis à ne pas communiquer avec la sphère publique dans une langue autre que le français ${ }^{54} »$.

De leur côté, les institutions et organisations non gouvernementales trouvent «inadmissibles les discriminations entre les communautés linguistiques fondées sur [...] leur degré de

${ }^{51}$ Déc. $94-345$ DC, cons. ${ }^{\circ} 6$

${ }^{5}$ Décret du 2 thermidor an II (20 juillet 1794) selon lequel «nul acte ne pourra, dans quelque partie que ce soit du territoire, être écrit qu'en langue française ».

53 Déc. $n^{\circ}$ 96-373 DC du 9 avril 1996, cons. n 91; JORF, 13 avril 1996, p. 5724.

54 Guy CARCASSONNE, «Etude sur la compatibilité entre la Charte européenne des langues régionales ou minoritaires et la Constitution», adressée au premier Ministre en septembre 1998, p. 36.

$\operatorname{PDLC} n^{\circ}$ 13, 2002 
souveraineté politique $[\ldots]^{55} »$, convenant ainsi de la réalité de «discriminations verticales». Ainsi, forgé autour de la langue nationale, le droit linguistique turc s'est caractérisé non seulement par le défaut d'incitations en faveur des langues infra-nationales, mais aussi par des dispositions contraignantes à leur encontre. La portée de la proscription de toute discrimination en raison de la langue, principe constitutionnel $^{56}$, est enserrée dans une conception rigide de l'unité nationale et d'intégration territoriale par le législateur et la Cour constitutionnelle. Celui-ci a interdit la modification par les partis politiques de la langue officielle turque ${ }^{57}$, l'affirmation de l'existence de minorités, notamment fondée sur des différences linguistiques ${ }^{58}$ et le déploiement d'une logique de protection, de promotion et de diffusion d'une langue ou d'une culture non turque dans le but de la destruction de l'intégrité nationale ${ }^{59}$. Celle-là a, le 14 juillet 1993 , dissous le parti politique kurde Özdep ${ }^{60}$ aux motifs de positions sécessionnistes et d'atteinte au principe de laïcité ${ }^{61}$ et d'affirmation politique de l'existence d'un peuple kurde distinct doté de culture et langue propres ${ }^{62}$.

L'officialisation par un Etat des langues infra-nationales qui y sont pratiquées constitue indubitablement l'acte de reconnaissance et le mode de traitement les plus aboutis. La volonté de consacrer à «toute communauté linguistique » «le droit de voir sa langue utilisée comme langue officielle dans son territoire ${ }^{63} »$, sachant que la langue «propre

${ }^{55}$ Art. 10 de la Déclaration universelle des droits linguistiques de juin 1996, in http://www.egt.ie/udhr/udlr-fr.html, p. 6.

${ }^{56}$ Art. $10 \S 1$ et $14 \S 1$ de la Constitution turque.

57 Art. 78 littera a de la loi $\mathrm{n}^{\circ} 2820$ portant réglementation des partis politiques.

${ }^{58}$ Idem, art. 81 littera a.

${ }^{59}$ Idem, art. 81 littera $\mathrm{b}$.

${ }^{60}$ CEDH, 8 décembre 1999, Aff. Parti de la liberté et de la démocratie c./Turquie, Req. $\mathrm{n}^{\circ} 00023885 / 94$, point $\mathrm{n}^{\circ} 14$.

${ }^{61}$ Idem, point $\mathrm{n}^{\circ} 9$.

${ }^{62}$ Idem, point $\mathrm{n}^{\circ} 38$

63 Art. $15 \S 1$ de la Déclaration universelle des droits linguistiques de juin 1996, préc., supra note $\mathrm{n}^{\circ} 55$, p. 6.

\section{$\operatorname{PDLC~} n^{\circ}$ 13, 2002}


d'un territoire » n'est pas forcément « la langue officielle d'Etat ${ }^{64} »$, a été manifestée. En Croatie, une loi de mai 2000 a posé le principe d'égalité en matière d'officialisation de la langue et de l'écriture des minorités nationales ${ }^{65}$. Dans ce cas, la recherche de l'égalité horizontale rejoint la perspective d'une égalité verticale plus prometteuse en terme d'encouragement des langues infra-nationales.

\section{La difficile élimination des « discriminations horizontales »}

Même s'il ne s'agit pas d'assurer «une égalité complète entre les langues ${ }^{66}$ », la notion de «discrimination horizontale » est mieux acceptée car, par définition, elle n'intéresse pas la langue d'Etat. Cette inégalité se manifeste par un tri des idiomes intra-étatiques à encourager. La «compréhension mutuelle entre tous les groupes linguistiques d'un Etat $^{67} \gg$ implique l'éviction d'une hiérarchisation entre langues sub-nationales. Des interactions seraient requises «dans les domaines couverts » par la Charte entre groupes distincts pratiquan une de ces langues «sous une forme identique ou proche» ou entre groupes d'un Etat pratiquant des langues régionales différentes ${ }^{68}$. Les langues intégrant la définition d'une «langue régionale ou minoritaire » doivent disposer d'un traitement juridique identique. A propos de leur situation et du statut juridique des locuteurs eux-mêmes, la clause de la mesure nationale la plus favorable, au moment de la ratification de la Charte dans le droit interne, doit être respectée ${ }^{69}$.

Selon le Conseil d'Etat dans son avis sur la compatibilité de la Charte avec la Constitution du 24 septembre 1996, « à condition que le soutien à l'expression dans ces langues dans les médias et sur le plan de l'action culturelle soit dévolu également à toutes les langues, au

${ }^{64}$ Idem, art. 6, p. 5.

${ }^{65}$ Application de la Charte européenne des langues régionales ou minoritaires à la Croatie, 20 septembre 2001, ECRML (2001) 2 Ann. II, Obs. de la Croatie, p. 37.

${ }^{66} \mathrm{http}: / /$ conventions.coe.int/treaty/fr/Reports/Html/148.htm, $\$ 72$, p. 10.

${ }^{67}$ Art. $7 \S 3$ de la Charte européenne des langues régionales ou minoritaires.

${ }^{68}$ Idem, art. 7 \& 1 lettre e).

${ }^{69}$ Idem, art. 4 § 2 .

$\operatorname{PDLC} n^{\circ} 13,2002$ 
sens de la Charte, se trouvant dans les mêmes conditions, la plupart des dispositions des articles 11 sur les médias et 12 sur la vie culturelle pourraient être mises en vigueur en France sans se heurter à une objection d'ordre constitutionnel ».

L'égalité horizontale s'exprime alors dans le soutien destiné «à toutes les langues, au sens de la Charte», placées «dans les mêmes conditions ».

Dans les Etats d'Europe comme dans d'autres continents, cette forme d'égalité inter-linguistique est faiblement appliquée, voire inexistante. Ainsi, même si la loi sur l'Institut canadien des langues patrimoniales de février 1991 plaça les langues indiennes sur le même pied d'égalité que les autres langues minoritaires non officielles du Canada, le français est à la fois langue officielle d'Etat et langue officielle du Québec. Dans le cadre de ses compétences (justice, éducation, santé, etc.), le Québec stimula l'emploi des langues minoritaires non officielles dans l'enseignement de groupes conventionnés. La Charte de la langue française de 1977 reconnaît aux Amérindiens et aux Inuits le «droit [...] de maintenir et de développer leurs langue et culture d'origine ${ }^{70} \gg$.

Aux Pays-Bas, l'officialisation territoriale du frison (province de Frise) conduit à des modalités particulières de promotion par rapport aux autres langues sub-étatiques, à l'instar des conventions liant l'administration provinciale et l'Etat ${ }^{71}$. Les actions en faveur des autres langues sub-étatiques sont moins appuyées ${ }^{72}$. L'encouragement modéré de la langue basse-saxonne dans la «vie publique» et dans la «vie privée $^{73} »$, les incitations culturelles concernant le limbourgeois dans la province du Limbourg ${ }^{74}$, l'absence de promotion du rom et du sinti ${ }^{75}$ et

${ }^{70}$ Pour plus d'informations, v. TRUDEL F., «Les langues autochtones au Québec », in http://www.clf.gouv.qc.ca/pubb133/b133ch4.html.

${ }^{11}$ Application de la Charte européenne des langues régionales ou minoritaires aux Pays-Bas, Rapport du 20 septembre 2001, ECRML (2001) 1, § 87, p. 8

${ }^{72}$ Idem, § 17 à 19 , p. 11.

${ }^{73}$ Idem, \& 21, p. 11

${ }^{74}$ Idem, \& 22, p. 12

${ }^{75}$ Idem, $\S 23$, p. 12

PDLC n ${ }^{\circ}$ 13, 2002 
la promotion du yiddish dans un cadre purement privé ${ }^{76}$ sont autant de traitements différenciés d'idiomes infra-nationaux.

En Finlande, autre illustration d'inégalité horizontale, le finnois et le suédois y sont les deux langues nationales ${ }^{77}$ officielles $^{78}$, usitées au Parlement, même si la minorité suédoise représente $6 \%$ de la population. Le principe du parallélisme des formes requiert une réponse dans une langue officielle identique à celle utilisée lors de la demande à l'administration, y compris en matière judiciaire. Aux îles Aaland, territoire finlandais présentant une caractéristique suédoise, le consentement de la municipalité concernée est nécessaire à l'enseignement du finnois dans les écoles primaires entretenues et subventionnées par l'Etat ou la municipalité. Le suédois y est la langue d'administration. Son emploi dans la «vie publique » répond à une double exigence de territorialité et de personnalité ${ }^{79}$. De plus, si la préservation des langues et cultures sâmes ${ }^{80}$ et romani a valeur constitutionnelle $^{81}$, le romani reste rarement utilisé, sauf dans les médias $^{82}$. Le tatar et le yiddish sont inexistants dans la «vie publique $»^{83}$. En matière de traitement subventionnel, l'aide publique à l'enseignement préscolaire au secondaire concerne le romani ${ }^{84}$. L'enseignement du yiddish et du $\operatorname{tatar}^{85}$, du russe en préscolaire ${ }^{86}$ et du sâme avec un soutien municipal dans ce dernier $\operatorname{cas}^{87}$ relève du privé.

${ }^{76}$ Idem, § 24, p. 12.

${ }^{77}$ Article 14 de la Constitution finnoise.

${ }^{78}$ Rapport du Comité des experts sur l'application de la Charte européenne des langues régionales ou minoritaires à la Finlande, 9 février 2001, § 10, p. 8

${ }^{79}$ Idem, § 19 , p. 9

${ }^{80} \mathrm{Idem}, \S 20$, p. 9

${ }^{81}$ Idem, § 44, p. 13

${ }^{82}$ Idem, \& 37, p. 12

${ }^{83}$ Idem, § 38, p. 12.

${ }^{84}$ Idem, § 44, p. 13.

${ }^{85}$ Idem, § 46 à 48, p. 13.

${ }^{86}$ Idem, § 49, p. 13.

${ }^{87}$ Idem, § 133, p. 29.

$\operatorname{PDLC~n}^{\circ}$ 13, 2002 
Sur le territoire sâme, le sâme est autorisé devant les autorités judiciaires avec le recours aux traductions et interprétations ${ }^{88}$. Les requêtes, preuves ${ }^{89}$ et actes officiels ${ }^{90}$ y sont recevables tant dans les procédures civiles $^{91}$ que devant les juridictions administratives ${ }^{92}$. La loi sur l'emploi du sâme s'applique aux autorités administratives d'Etat ${ }^{93}$ et du territoire sâme ${ }^{94}$. Les langues des Skolttes et d'Irani sont menacées d'extinction ${ }^{95}$.

L'association entre les réalités institutionnelles et linguistiques peut être à la source des inégalités horizontales. La structure fédérale de la Belgique et de l'Allemagne y participe grandement. Mais, les inégalités se retrouvent aussi dans les Etats unitaires. En sens contraire, certains Etats régionalisés tendent vers la non-discrimination horizontale entre langues territoriales, à l'instar de l'Espagne.

A défaut de langue commune à l'ensemble de ses citoyens, la Belgique ne connaît que des antagonismes horizontaux résultant de la tendance régionale à une "assimilation des minorités en milieu linguistique ${ }^{96}$, malgré les rivalités inter-linguistiques intra-régionales. Dans les Communautés, la connaissance de la langue régionale est une condition d'accès à une fonction ou un emploi ${ }^{97}$, forme « d'affirmative action » basée sur un critère valorisant cette langue. Au titre de l'unilinguisme régional, elle est employée dans les services locaux et

\author{
${ }^{88} \mathrm{Idem}, \S 144$, p. 32. \\ ${ }^{89}$ Idem, § 147, p. 32. \\ ${ }^{90}$ Idem, \$ 148 , p. 33 . \\ ${ }^{91}$ Idem, $\S 149$, p. 33. \\ ${ }^{92}$ Idem, $\$ 154$, p. 33. \\ ${ }^{93} \mathrm{Idem}, \S 156$, p. 34. \\ ${ }^{94}$ Idem, § 158 , p. 34. \\ ${ }^{95}$ Recommandations du Comité des ministres du Conseil de l'Europe sur \\ l'application de la Charte pour la Finlande, $\S 1$. \\ 96 V. position de la Commission européenne des droits de l'homme, CEDH, \\ 23 juillet 1968, Aff. relative à certains aspects du régime linguistique de \\ l'enseignement en Belgique, point $\mathrm{n}^{\circ} 12$, préc., supra note $\mathrm{n}^{\circ} 10$. \\ ${ }^{97}$ Art. 15 de la loi du 18 juillet 1966 sur l'emploi des langues en matière \\ administrative coordonnée.
}

\title{
$\operatorname{PDLC~} n^{\circ}$ 13, 2002
}


les services intérieurs notamment ${ }^{98}$ et pour les services régionaux dont les avis, communications et formulaires destinés au public, les actes, les certificats, les déclarations et les autorisations concernant les (ou délivrés aux) particuliers sont rédigés dans cette langue ${ }^{99}$.

Les exceptions à l'unilinguisme régional concernent les communes à facilités de la frontière linguistique, Bruxelles-Capitale, la Communauté germanophone et les actes des Communautés. Premièrement, les communes à facilités ont un régime linguistique spécial garantissant l'emploi d'une langue autre que celle de la région linguistique où elles sont situées. La Communauté doit y assurer le droit d'offrir et de recevoir des services dans la langue de la Communauté limitrophe, même si elle est minoritaire dans les communes concernées ${ }^{100}$. Deuxièmement, à Bruxelles-Capitale, malgré une minorité flamande, le bilinguisme s'impose aux organismes du gouvernement fédéral et de cette région. La langue d'enseignement y est le français ou le néerlandais selon la langue maternelle ou usuelle de l'enfant ${ }^{101}$. Troisièmement, dans la Communauté germanophone, l'allemand et le français sont les langues de rédaction des avis, communications et formulaires communaux destinés au public ${ }^{102}$. Enfin, les Communautés de Belgique ont un pouvoir normatif en matière linguistique ${ }^{103}$.

98 Art. 10 de la loi du 18 juillet 1966 sur l'emploi des langues en matière administrative coordonnée.

${ }^{99}$ Idem, art. 33.

${ }^{100}$ Communauté française de Belgique,

http://www.ciral.ulaval.ca/alx/amlxmonde/europe/belgiquefrn.htm, p. 5.

${ }^{01}$ Art. 5 de la loi du 30 juillet 1963 relative au régime linguistique de l'enseignement.

${ }^{102}$ Art. $1 \S 2$ de la loi du 18 juillet 1966 sur l'emploi des langues en matière administrative coordonnée, conforté par l'article 12 alinéa 2.

${ }^{103}$ Pour la Communauté française de Belgique, décret de 1990 reconnaissant les «langues régionales endogènes » (wallon, picard, lorrain, champenois, luxembourgeois) et décret du 12 juillet 1978 sur la défense de la langue française.

$\operatorname{PDLC} n^{\circ}$ 13, 2002 
L'Allemagne illustre a priori une unité linguistique autour de l'Allemand même si, par amendement de 1994 du Grundsgesetz du 8 mai 1949, l'article $3 \S 3$ interdit toute discrimination en raison de la langue $^{104}$. Mais le traitement des langues sub-nationales varie d'un Land à l'autre. En Basse-Saxe, le frison oriental est enseigné facultativement en préscolaire et en primaire et est toléré dans les médias $^{105}$. Dans le Land de Schleswig-Holstein, l'article 5 de la Constitution reconnait le droit au soutien et à la protection de la «minorité nationale danoise » et du «groupe ethnique des Frisons ${ }^{106}$. Sur la base de la Déclaration du gouvernement de ce Land du 29 septembre 1949 et de l'entente bilatérale entre l'Allemagne et le Danemark, le traitement de la minorité danoise d'Allemagne dépend de celui de la minorité allemande au Danemark ${ }^{107}$. L'usage du danois est encouragé devant les tribunaux pour les personnes dans l'impossibilité d'utiliser l'allemand ${ }^{108}$, devant les pouvoirs publics, en matière de radiodiffusion et d'enseignement ${ }^{109}$ facultatif en vertu de l'article 8 § 4 de la Constitution de ce Land $^{110}$. Par contre, dans le Land de Brandebourg, le sorabe est langue co-officielle du fait de l'insertion dans les droits acquis de la loi protectrice des droits de la population sorabe de 1948 dans l'ex-République démocratique allemande, de l'article 35 § 3 du Traité d'union entre les Allemagne du 11 août 1990 sur la liberté de préserver et de parler sa langue «dans la vie publique » et de l'article 25 de la Constitution du Land garantissant la préservation, la protection et la promotion de l'identité nationale sorabe et des zones de peuplement traditionnelles. La co-

\footnotetext{
${ }^{104}$ Land de Basse-Saxe,

http://www.ciral.ulaval.ca/alx/amlxmonde/EtatsNsouverains/basse-saxe.htm, p. 3

${ }^{105}$ Idem, p. 5

${ }^{106}$ Land de Schleswig-Holstein,

http://www.ciral.ulaval.ca/alx/amlxmonde/EtatsNsouverains/schleswig-

Holstein.htm, p. 6.

${ }^{107}$ Idem, p. 6

${ }^{108}$ Idem, p. 7.

${ }^{109}$ Idem, p. 6.

${ }^{110}$ Idem, p. 8.
}

$\operatorname{PDLC~n}^{\circ}$ 13, 2002 
officialisation, circonscrite dans les districts reconnus par cet Etat fédéré ${ }^{111}$, est confortée par le «droit d'usage » du sorabe devant les tribunaux sis dans ces zones et par l'obligation pour le juge nonlocuteur de recourir à un interprète ${ }^{12}$.

Ces exemples non exhaustifs montrent que l'égalité horizontale souhaitée par la Charte européenne n'est pas acquise face aux réalités politiques, sociales, historiques, linguistiques, juridiques et institutionnelles. Toutefois, les contraintes dans l'application des principes devant l'objectif de promotion de ces idiomes sont plus larges.

\section{II. - $\quad$ LES CONTRAINTES DANS L'APPLICATION DES PRINCIPES ET LA PROMOTION DES LANGUES INFRA-ETATIQUES}

Les divers aspects d'un même principe entraînent des contraintes autorégulatrices dans l'encouragement des langues infraétatiques (A). Les limites promotionnelles des principes du droit amènent la problématique sur le terrain de leur conservation, de leur modernisation et de leur dépassement simultanés par l'invocation d'un statut avancé de ces langues (B).

\section{A. - L'encouragement des langues sub-étatiques par autorégulation des principes du droit}

L'autorégulation concerne les principes de liberté (1) et d'égalité (2)

${ }^{111}$ Land de Brandebourg,

http://www/ciral.ulaval.ca/alx/amlxmonde/EtatsNsouverains/brandebourg.htm pp. 4-6.

Idem, p. 7

$\operatorname{PDLC~n}^{\circ}$ 13, 2002 


\section{La promotion des langues infra-étatiques par la conciliation} des libertés

La mission de protection des identités linguistiques régionales et minoritaires a été laissée aux Etats ${ }^{113}$. Cette considération conduit à s'interroger notamment sur la contribution des collectivités territoriales au pluralisme linguistique.

Une conciliation des libertés autorise une organisation territoriale de l'encouragement des langues régionales ou minoritaires. Il s'agit de promouvoir, selon les Etats, à divers degrés, la liberté individuelle ou collective et les libertés locales. Le fait pour la Conférence permanente des pouvoirs locaux et régionaux de l'Europe d'être à l'origine de la Charte «en raison du rôle que doivent être amenés à jouer les collectivités territoriales ${ }^{114} »$, a contribué à ce que ce traité soutienne la promotion de ces langues par ces collectivités dans le cadre de l'administration régionale ou locale ${ }^{115}$ (présentation des demandes orales ou écrites ${ }^{116}$, publication des textes officiels des collectivités régionales ${ }^{117}$ et des collectivités locales ${ }^{118}$, emploi ou adoption, le cas échéant conjointement avec la dénomination dans la (les) langue(s) officielle(s), des formes traditionnelles et correctes de la toponymie $\left.{ }^{119}\right)$. Leur usage dans ces collectivités n'exclut pas le recours à une (ou aux) langue(s) officielle(s) ${ }^{120}$.

${ }^{113}$ Déclaration des droits de personnes appartenant à des minorités nationales ou ethniques, religieuses et linguistiques, résolution $n^{\circ} 47 / 135$ du 18 décembre 1992 de l'Assemblée générale des Nations-Unies.

$114 \mathrm{http}: / /$ conventions.coe.int/treaty/fr/Reports/Html/148. htm, § 5, p. 1 .

115 Art. $10 § 2$ lettre a) de la Charte européenne des langues régionales ou minoritaires.

${ }^{116} \mathrm{Idem}$, art. 10 \& 2 lettre b)

${ }^{117}$ Idem, art. $10 \& 2$ lettre c)

${ }^{118}$ Idem, art. $10 \S 2$ lettre d).

${ }^{119}$ Idem, art. $10 \S 2$ lettre g).

120 Pour les collectivités régionales, art. $10 \$ 2$ lettre e) de la Charte européenne des langues régionales ou minoritaires; pour les collectivités locales, idem, art. $10 \S 2$ lettre f).

\section{$\operatorname{PDLC~n}^{\circ}$ 13, 2002}


En France, l'extension sectorielle et la généralisation de la promotion des langues et cultures régionales ont accru le champ d'intervention des collectivités territoriales en la matière. L'emprise étatique sur la définition des programmes d'enseignement et de formation, premier domaine de l'expression du pluralisme idiomatique, tendait à réduire les actions locales. Cependant, l'encouragement par une collectivité territoriale de l'enseignement d'une langue et d'une culture régionales ne porte atteinte, sous certaines conditions, à aucun principe à valeur constitutionnelle ${ }^{121}$. Les Conseils régionaux d'outremer peuvent développer des activités éducatives et culturelles portant sur leur connaissance et susceptibles d'être organisées dans les établissements scolaires relevant de leur compétence ${ }^{122}$. Les activités «complémentaires » et «facultatives » financées ne portent pas atteinte et ne se substituent pas aux programmes d'enseignement et de formation définis par l'Etat. Ces collectivités territoriales élaborent un «programme culturel régional, notamment dans le domaine des langues régionales ${ }^{123} »$.

L'action des collectivités territoriales repose sur des modalités unilatérales (plan de développement d'un enseignement de la langue et de la culture régionales ${ }^{124}$ ) et bilatérales (convention entre une collectivité territoriale et l'Etat pour l'insertion de leur enseignement dans le temps scolaire dans le cadre dudit $\operatorname{plan}^{125}$, conventions entre la collectivité territoriale et les sociétés publiques audiovisuelles pour réaliser des programmes TV et de radiodiffusion dont l'objet est le développement de la langue et de la culture régionales pour une

${ }^{121}$ Déc. 91-290 DC du 9 mai 1991, cons. n 37 ; JORF, 14 mai 1991, p. 6350. ${ }^{122}$ Art. L. 4433-25 CGCT tel que résultant de l'art. 21 de la loi $\mathrm{n}^{\circ}$ 84-747 du 2 août $1984 ; J O R F, 3$ août 1984, p. 2559.

Art. L. 4433-27 CGCT résultant de l'art. 23 de la loi n $84-747$ du 2 août 1984.

${ }^{124}$ Art. 23 de la loi n ${ }^{\circ}$ 2001-616 du 11 juillet 2001 relative à Mayotte, préc., supra note $\mathrm{n}^{\circ} 39$.

${ }^{125}$ Art. L. 4424-14 CGCT tel que résultant de l'art. 53 de la loi n ${ }^{\circ} 91-428$ du 13 mai 1991 portant statut de la collectivité territoriale de Corse ; JORF, 14 mai 1991 , p. 6.318.

$\operatorname{PDLC} n^{\circ} 13,2002$ 
diffusion circonscrite à une région ${ }^{126}$ ). La concertation interinstitutionnelle n'est pas exclue. En matière d'aménagement du territoire, le «schéma de services collectifs culturels », élaboré par l'Etat avec la collaboration des collectivités territoriales notamment ${ }^{127}$ a, entre autres, la mission de déterminer des «actions à mettre en œuvre pour assurer [...] la sauvegarde des langues régionales ou minoritaires $^{128} »$.

Un ensemble de pays opte, en raison de leur structure institutionnelle, pour le principe de territorialité. En Espagne, les statuts d'autonomie déterminent le caractère officiel des langues régionales dans les communautés autonomes respectives, assimilées à des «langues espagnoles ${ }^{129}$ ». L'officialisation des langues régionales s'exprime aussi dans les lois de normalisation linguistique ${ }^{130}$. La qualité de «langue propre » régionale emporte un usage public de jure à tous les niveaux dans les relations avec l'administration d'Etat ${ }^{131}$ et une liberté de choix entre langues officielles lorsque les citoyens de la Communauté concernée s'adressent à elle.

En Allemagne, l'organisation du droit linguistique dépend de la législation des Länder, sans aller à l'encontre des lois fédérales ${ }^{132}$. En

\footnotetext{
${ }^{126}$ Art. L. 4424-16 CGCT tel que résultant de l'art. 55 de la loi ${ }^{\circ} 91-428$ du 13 mai 1991 ; art. L 3551-2 CGCT tel que prévu à l'art. 23 de la loi n 2001 616 du 11 juillet 2001 relative à Mayotte, préc., supra note $n^{\circ} 40$.

127 Art. 11 de la loi $\mathrm{n}^{\circ} 99-533$ du 25 juin 1999 d'orientation pour l'aménagement du territoire et le développement durable du territoire et portant modification de la loi $\mathrm{n}^{\circ} 95-115$ du 4 février 1995 d'orientation pour l'aménagement et le développement du territoire; JORF, 29 juin 1999 , p. 9.515 .

${ }^{28}$ Idem, art. $14 \S 2$.

${ }^{129}$ Art. 3 de la Constitution espagnole ; Art. 20 du statut d'autonomie basque de 1979 selon lequel «le basque est la langue officielle de la patrie basque à tous les niveaux et sur toute l'étendue de son territoire [...] ».

${ }^{130}$ Préambule de la loi 1/1998 du 7 janvier 1998 sur la politique linguistique catalane in http://cultura.gencat.es/llengat/legis/LOH-1998-HTM, p. 1.

${ }_{131}$ Idem, p. 3.

${ }^{132}$ Land de Basse-Saxe, préc., supra note n ${ }^{\circ} 104$, p. 2.
}

\section{$\operatorname{PDLC~n}^{\circ}$ 13, 2002}


Finlande, la promotion de la langue et culture sâmes résulte d'une « compétence générale de surveillance » du parlement sâme ${ }^{133}$.

En Belgique, la structure fédérale de l'Etat belge tient compte des réalités linguistiques dominantes. Les trois communautés linguistiques, française, flamande et germanophone ${ }^{134}$ constituent des Etats fédérés compétents notamment en matière culturelle et d'enseignement ${ }^{135}$. Il y existe quatre régions linguistiques, de langue française, néerlandaise, allemande et bilingue de Bruxelles-Capitale ${ }^{136}$. Le principe de territorialité suppose une régionalisation normative de l'emploi des langues «à l'exclusion du législateur fédéral » en matière administrative ${ }^{137}$, pour l'enseignement dans les établissements créés, subventionnés ou reconnus par les pouvoirs publics ${ }^{138}$ et les relations sociales entre employeurs et leur personnel, les actes et documents d'entreprises imposés par la loi et les règlements ${ }^{139}$.

Les libertés locales s'exercent également dans le cadre de l'action internationale. La correspondance de certaines langues infraétatiques à une langue de culture nationale ou infra-étatique d'un autre Etat confère une importance singulière à la «collaboration transnationale $\mathrm{e}^{140} »$. Il n'est pas interdit, «dans l'intérêt des langues régionales ou minoritaires » au sens de l'article 14 littera b de la Charte, qu' « à travers les frontières, notamment entre collectivités territoriales ou locales sur le territoire desquelles la même langue est pratiquée de façon identique ou proche », puisse être facilitée ou promue une coopération.

${ }^{133}$ Rapport du Comité des experts sur l'application de la Charte européenne des langues régionales ou minoritaires à la Finlande, 9 février 2001, § 142, p. 31 .

${ }_{134}$ Art. 2 de la Constitution du 17 février 1994

${ }^{135}$ Idem, art $127 \S 1$ pour les communautés française et néerlandaise et art. $130 \S 1$ pour la communauté germanophone.

${ }^{136}$ Idem, art. 4.

${ }^{137} \mathrm{Idem}$, art. $129 \S 1$, point $\mathrm{n}^{\circ} 1$.

${ }^{138}$ Idem, art. $129 \S 1$, point $\mathrm{n}^{\circ} 2$

${ }^{139}$ Idem, art. $129 \S 1$, point $\mathrm{n}^{\circ} 3$.

${ }^{140}$ http://conventions.coe.int/treaty/fr/Reports/Html/148.htm, § 69, p. 10.

PDLC n ${ }^{\circ}$ 13, 2002 
Cet intérêt résulte aussi de la conciliation entre libertés sectorielles. La Cour de justice des Communautés européennes a sanctionné, au titre de la liberté d'établissement et de prestation de service, l'interdiction faite aux ressortissants d'autres Etats membres de créer des frontistiria ${ }^{141}$ pour l'apprentissage de langues étrangères. Cette jurisprudence rappelée par la Cour européenne des droits de l'homme ${ }^{142}$ peut concerner l'enseignement d'une langue régionale ou minoritaire au sens de l'article $1^{\text {er }}$ de la Charte. Le droit communautaire n'interdit pas aux Etats membres de l'Union européenne d'insérer dans les objectifs de service public la présentation des programmes destinés à des minorités, sans toutefois justifier des atteintes aux libertés économiques à ce titre ${ }^{143}$. La nécessité de garantir le pluralisme de la presse écrite flamande ne fonde pas, conformément au principe de proportionnalité, la limitation au principe de liberté d'établissement ${ }^{144}$.

Le rôle des pouvoirs publics en matière de radiotélévision est de stimuler, sur les territoires où ces langues sont pratiquées, selon la situation de chacune d'elles, leur usage dans le respect des principes d'indépendance et d'autonomie des médias ${ }^{145}$. Il peut s'agir d'adapter le service public (création d'au moins une station de radio et une chaîne de télévision dans ces langues ${ }^{146}$, prise de dispositions particulières pour la programmation des émissions dans ces langues ${ }^{147}$ ), garantir la liberté de communication dans le privé ${ }^{148}$, jointe éventuellement à la liberté d'entreprise (création d'au moins une

141 CJCE, 19 mars 1988, Aff. n 147/86, Commission c./République hellénique, Rec. 1988, p. 1637.

142 CEDH, 19 mars 1997, Aff. Hornsby c./Grèce, Req. n 00018357/91, Rec. 1997-II, p. 152.

${ }^{143}$ TPI, 10 juillet 1991, Aff. T-69/89, RTE c./Commission, Rec. 1989, p. II-48

144 Déc. Commission du 26 juin 1997 au titre de l'art. 90 \& 3 du traité CE concernant le droit exclusif d'émettre de la publicité télévisée en Flandre, (97/606/CE), point n ${ }^{\circ} 13 ; J O C E \mathrm{n}^{\circ}$ L. 244 du 6 septembre 1997, p. 17.

${ }_{145}$ Art. $11 \S 1$ de la Charte européenne des langues régionales ou minoritaires.

${ }^{146} \mathrm{Idem}$, art. $11 \S 1$ lettre a), i.

${ }^{147}$ Idem, art. $11 \S 1$ lettre a), iii.

${ }^{148}$ Idem, art. $11 \S 1$ lettre b), ii.

PDLC n ${ }^{\circ}$ 13, 2002 
station de radio ou d'une chaîne de télévision ${ }^{149}$, production et diffusion d'œuvres audio ou audiovisuelles ${ }^{150}$, création et/ou le maintien d'au moins un organe de presse ${ }^{151}$ ou la publication régulière d'articles de presse ${ }^{152}$ dans ces langues). Les Etats doivent éviter d'entraver la création et l'utilisation de médias écrits par les personnes appartenant à des minorités ${ }^{153}$, sachant que les mesures législatives peuvent être utiles au «pluralisme culturel ${ }^{154}$ ». Le recours à ces langues devrait accompagner l'industrie et l'administration de la culture, sous toutes ces formes ${ }^{155}$, y compris en s'appuyant sur les nouvelles technologies.

L'humanisme qui gouverne le traitement des langues au titre des droits de l'homme préside à leur promotion et/ou à leur protection en référence aux droits fondamentaux ou aux principes du droit. Le droit de les pratiquer doit se conformer «à l'esprit de la Convention de sauvegarde des droits de l'homme et des libertés fondamentales ». Les dispositions de la Charte sur les médias rappellent l'article 10 de cette Convention garantissant la liberté d'opinion, de réception ou de communication des informations ou des idées, sans ingérence d'autorités publiques et sans considération de frontière ${ }^{156}$ et ce, dans le respect de l'indépendance et de l'autonomie des médias par rapport aux pouvoirs publics ${ }^{157}$. Le principe de liberté d'expression et de circulation de l'information est encadré dans l'esprit et les termes de l'article $10 \S 2$ de la CEDH, conformément à l'article $11 \S 2$ de la Charte.

${ }^{149}$ Idem, art. $11 \S 1$ lettre b), i.

${ }^{150}$ Idem, art. $11 \S 1$ lettre d)

${ }^{151} \mathrm{Idem}$, art. $11 \S 1$ lettre e),

${ }^{152}$ Idem, art. $11 \S 1$ lettre e), ii.

153 Art. 9 \$3 de la Convention-cadre sur la protection des minorités nationales.

${ }^{154}$ Idem, art. 9 § 4

${ }^{155}$ Art. $12 \S 1$ de la Charte européenne des langues régionales ou minoritaires.

${ }^{156}$ Idem, art. $11 \S 2$.

${ }^{157}$ Idem, art. 11 § 1 .

PDLC n ${ }^{\circ}$ 13, 2002 
Enfin, garantir l'usage des langues sub-étatiques peut s'avérer utile à l'approfondissement de la liberté de commerce. Si les autorités publiques en ont la compétence et dans la mesure du raisonnablement possible, les Parties à la Charte s'engagent à définir, grâce à leurs règles financières et bancaires, des modalités permettant, dans des conditions compatibles avec les usages commerciaux, leur emploi dans la rédaction des ordres de paiement (chèques, traites, etc.) ou d'autres documents financiers $^{158}$, voire dans le secteur public ${ }^{159}$.

\section{Les égalités au service des langues infra-étatiques}

L'égalité-neutralité constitue une exigence minimale ${ }^{160}$. Elle est traduite par exemple en matière d'accès aux apprentissages de la langue sub-étatique des locuteurs ou non vivant dans une aire d'influence $^{161}$. Si la Cour européenne des droits de l'homme est amenée à connaître des différences de traitement basées sur la langue et l'appartenance à une minorité nationale dans le cadre de la $\mathrm{CEDH}^{162}$, la Charte sollicite la levée de barrières au recours aux langues infraétatiques. La structure administrative existante ou à établir ne doit pas être un obstacle à leur promotion ${ }^{163}$ à travers l'utilisation du service public ou l'exercice de prérogatives de puissance publique.

La Charte pose la «nécessité d'une action résolue » en raison de sa fonction de sauvegarde ${ }^{164}$ pour inciter toutes formes d'expressions linguistiques, tant orale qu'écrite et tant «dans la vie publique » que « dans la vie privée ${ }^{165} »$.

\footnotetext{
${ }^{158}$ Art. $13 \S 2$ lettre a) de la Charte européenne des langues régionales ou minoritaires.

${ }^{159}$ Idem, art. $13 \S 2$ lettre b)

160 http://conventions.coe.int/treaty/fr/Reports/Html/148.htm, § 71, p. 10.

${ }^{161}$ Art. 7 \& 1 lettre g) de la Charte européenne des langues régionales ou minoritaires.

162 CEDH, 2 mars 1987, Mathieu MOHIN

points $\mathrm{n}^{\circ} 58$ et 59 , préc., supra note $\mathrm{n}^{\circ} 10$.

${ }^{6}$ Art. 7 \$ 1 lettre b) de la Charte européenne des langues régionales ou minoritaires.

${ }^{164}$ Idem, art. $7 \S 1$ lettre c).

${ }^{165} \mathrm{Idem}$, art. 7 § 1 lettre d)
}

\section{$\operatorname{PDLC~} n^{\circ}$ 13, 2002}


Face aux oppositions notables entre les partisans et les détracteurs de l'existence de «discriminations verticales », un consensus médian se situe dans l'observation des «disparités entre la situation des langues officielles et celle des langues régionales ou minoritaires », ces dernières étant «souvent désavantagées ${ }^{166}$ ». La «correction des déséquilibres linguistiques ${ }^{167}$ » requiert des «affirmative actions » ratione linguae. Cette conception ne peut être niée dès lors que le Conseil de l'Europe renvoie à une application souple des quotas requis pour constituer une classe, à savoir qu'un nombre inférieur d'élèves peut être «jugé suffisant ${ }^{168} »$. La discrimination positive répond à deux fonctions, garantir l'égalité entre les locuteurs et le reste de la population et considérer leurs situations particulières au titre de l'adaptation ${ }^{169}$. «Les préférences linguistiques » en matière d'enseignement étaient exclues du catalogue des droits et libertés garantis par la $\mathrm{CEDH}^{170}$.

En France, dans le secteur audiovisuel, il existe une obligation de consacrer, en particulier aux heures de grande écoute, des «proportions au moins égales à $40 \%$ à des œuvres d'expression originale française » dans la diffusion d'œuvres cinématographiques incluses dans le cahier des charges des sociétés nationales, les

${ }^{166}$ http://conventions.coe.int/treaty/fr/Reports/Html/148.htm, § 73, p. 10

${ }_{167}$ Cons. $\mathrm{n}^{\circ} 17$ de la Déclaration universelle des droits linguistiques de juin 1996, in http://www.egt.ie/udhr/udlr-fr.html, p. 2

${ }_{168}$ http://conventions.coe.int/treaty/fr/Reports/Html/148.htm, § 82, pp. 11-12.

169 Voir la.position de la Finlande à propos du sâme in Application de la Charte européenne des langues régionales ou minoritaires en Finlande, Rapport du Comité d'experts, 20 septembre 2001, ECRML (2001) 3, § 177, p. 38. La similitude des critères d'attribution de subventions à la production et à la distribution des œuvres audio et audiovisuelles en langues sâme et finnoise n'empêche pas d'adopter, «le cas échéant, la stratégie de la discrimination positive ».

${ }^{170} \mathrm{CEDH}, 23$ juillet 1968 sur le régime linguistique de l'enseignement en Belgique, point $\mathrm{n}^{\circ} 7$, préc., supra note $\mathrm{n}^{\circ} 10$.

$\operatorname{PDLC} n^{\circ}$ 13, 2002 
autorisations et les décrets ${ }^{171}$. Les œuvres cinématographiques ou audiovisuelles d'expression originale française sont «réalisées intégralement ou principalement en version originale en langue française ou dans une langue régionale en usage en France ${ }^{172} »$.

Il s'agit d'une «affirmative action » à vocation générale née de la rencontre entre l'hégémonie de l'art cinématographique des EtatsUnis et la logique intégrative française touchant à la fois les langues nationale et régionales. Cette exception culturelle n'entraîne pas le bénéfice par un parler local de l' «affirmative action » vis-à-vis du français. Le quota sus-évoqué concerne en pratique plus largement les œuvres de langue et culture françaises, réserve faite des sociétés de programmes de télédiffusion assurant la promotion des langues et des cultures régionales du fait de leur vocation régionaliste ${ }^{173}$.

La faiblesse de capacités des productions culturelles par le truchement des langues infra-étatiques ${ }^{174}$ due à une assise démographique limitée et aux difficultés de réaliser des économies d'échelle, rend le soutien public indispensable pour stimuler l'expression et les initiatives qui leur sont propres et favoriser l'accès aux œuvres produites dans ces langues ${ }^{175}$. L'inter-traduction des œuvres doit être assurée ${ }^{176}$. Le déploiement des activités culturelles en intégrant la pratique d'une (ou des) langue(s) régionale(s) ou

${ }^{171}$ Art. 70 de la loi n ${ }^{\circ} 86-1067$ du 30 septembre 1986 relative à la liberté de communication ; JORF, $1^{\text {er }}$ octobre 1986, p. 11.755

2 Déc. $n^{\circ} 98-D-70$ du Conseil de la concurrence du 24 novembre 1998 relative à la saisine des sociétés multivision et télévision par satellite (TPS) dans le secteur des droits de diffusion audiovisuelle; BOCCRF, 21 janvier 1999, p. 7, spéc. p. 9.

173 Concernant RFO, art. 4 de la loi $\mathrm{n}^{\circ} 2000-719$ du $1^{\mathrm{er}}$ août 2000 modifiant l'art. 44 de la loi n $86-1067$ du 30 septembre 1986 relative à la liberté de communication ; JORF, 2 août 2000, p. 11.903.

${ }_{174}$ http://conventions.coe.int/treaty/fr/Reports/Html/148.htm, § 116, p. 16.

175 Art. 12 \& 1 lettre a) de la Charte européenne des langues régionales ou minoritaires.

${ }^{176}$ Pour la traduction des œuvres produites en langues sub-étatiques dans d'autres langues, idem, art. $12 \S 1$ lettre b) ; et inversement, idem, art. $12 \S 1$ lettre c)

\section{$\operatorname{PDLC~} n^{\circ}$ 13, 2002}


minoritaire(s) ${ }^{177}$ implique la mise à la disposition d'un personnel la (ou les) maîtrisant, en plus de la (des) langue(s) du reste de la population, auprès des organismes responsables de ces activités culturelles ${ }^{178}$.

\section{B. - Le dépassement des principes dans le traitement de langues sub-étatiques}

L'analyse des répercussions juridiques de la mise en œuvre des principes du droit en matière idiomatique révèle quelques limites (1). Devant les menaces pesant sur certaines langues infra-nationales, l'amélioration de leur statut juridique a été préconisé (2).

1. La contribution des conflits téléologiques d'autorégulation et « inter-principes » aux restrictions d'usage idiomatique

La promotion des langues sub-étatiques est canalisée par les relations « intra-principes ». Les rapports entre la liberté d'appréciation d'un tribunal et la liberté de choix de la langue est traitée de deux façons. La priorité donnée à la préservation des droits des minorités, y compris dans leurs liens avec la justice, par la Convention-cadre suppose le «droit de toute personne appartenant à une minorité d'être informée [...] dans une langue qu'elle comprend des raisons de son arrestation, de la nature et de la cause de l'accusation portée contre elle ainsi que de se défendre dans cette langue $[\ldots]^{179} \gg$ sans se rapporter à la liberté d'appréciation du tribunal. Par sa fonction de sauvegarde des langues régionales ou minoritaires, la Charte traite du rapport à la langue dans les tribunaux et en soumet le recours à la liberté d'appréciation du juge, responsable de la bonne administration de la justice $^{180}$. La gratuité des traductions et interprétations tant aux plans

${ }^{177}$ Idem, art. $12 \S 1$ lettre d)

${ }^{178}$ Idem, art. $12 \S 1$ lettre e).

179 Art. $10 \$ 3$ de la Convention-cadre sur la protection des minorités nationales.

${ }^{180}$ Art. $9 \S 1$ de la Charte européenne des langues régionales et minoritaires.

$\operatorname{PDLC~n}{ }^{\circ}$ 13, 2002 
pénal que civil ${ }^{181}$ assure une conciliation meilleure des libertés et atténue la prévalence des exigences de police prétorienne.

Les rapports entre les libertés économiques et la liberté de choix de la langue dans le cadre du droit communautaire réduisent l'encouragement tant des langues nationales que régionales. La «langue facilement comprise » par l'acheteur utilisée dans les mentions obligatoires d'étiquetage des produits alimentaires destinés à être vendus en l'état au consommateur en vertu de l'article 14 alinéa 2 de la directive $\mathrm{n}^{\circ}$ 79/112/CEE du 18 décembre 1978 n'équivaut ni à la «langue officielle de l'Etat», ni à la «langue de la région ${ }^{182}$ ». L'obligation d'appeler à une langue déterminée ${ }^{183}$, comme le recours exclusif à une langue d'une région linguistique ${ }^{184}$ ou à une langue d'un Etat membre où le produit est commercialisé est une restriction quantitative aux importations ${ }^{185}$, sauf justification tirée de l'information du consommateur ${ }^{186}$. Les exigences linguistiques sont des limitations aux libertés économiques ${ }^{187}$. La Charte garantit l'accès dans les langues sub-étatiques des informations fournies par les autorités compétentes sur le droit des consommateurs ${ }^{188}$.

La simple application de la «conception classique des libertés » conduit à la licéité de certaines limitations à l'emploi des langues infraétatiques. La jurisprudence européenne relative aux langues principales régionales est transposable aux cas de langues d'Etat. Parmi les

${ }^{181}$ Idem, art. $9 \S 1$ lettre d), art. $9 \S 1$ lettre b), ii et art. $9 \S 1$ lettre c), ii, http://conventions.coe.int/treaty/fr/Reports/Html/148.htm, $\S 96$, p. 13.

182 CJCE, 12 octobre 1995, Aff. C-85/94, Piageme/Peeters, point $n^{\circ} 15$, Rec. 1995, p. I-2955.

${ }^{183}$ Idem, point $\mathrm{n}^{\circ} 18$

${ }^{184} \mathrm{Idem}$, point $\mathrm{n}^{\circ} 17$.

${ }^{185}$ CJCE, 18 juin 1991, Aff. C-369/89, Piageme, Rec. 1991, p. 2971.

186 CJCE, 4 août 1994, Aff. C-5193, Meyhui NV et Scott Zwiesel Glasswerk AG, Rec. 1994, p. 3879.

${ }^{187}$ Communication interprétative de la Commission concernant l'emploi des langues pour la négociation des denrées alimentaires; JOCE $n^{\circ}$ C. 345 , 23 décembre 1993, p. 3.

${ }^{188}$ Art. 13 \& 2 lettre e) de la Charte européenne des langues régionales ou minoritaires.

\section{$\operatorname{PDLC~} n^{\circ}$ 13, 2002}


restrictions admises, il y a le refus de créer ou de subventionner des écoles dont la langue d'enseignement n'est pas la langue principale si, à côté d'un enseignement officiel ou subventionné, il existe un enseignement «libre »dans une langue minoritaire ${ }^{189}$, ou le retrait de subventions aux écoles dont la langue d'enseignement est une langue minoritaire à l'intérieur d'une région linguistique si son instruction n'est pas empêchée dans une école privée non subventionnée ou dans une école d'une région où cette langue est promue ${ }^{190}$.

La «conception classique des libertés » ressortant du Pacte international relatif aux droits civils et politiques des Nations-Unies réduit aussi la portée de la promotion des langues sub-étatiques. Dans l'affaire Mc Intyre et autres c./Canada, le Comité des droits de l'homme des Nations-Unies avait à examiner la proscription faite aux citoyens anglais du Québec d'utiliser l'anglais dans la publicité. Interprétant largement l'article $19 \S 2$ de ce Pacte, il souligna que «l'élément commercial d'une forme d'expression [...] ne peut avoir pour effet de faire sortir celle-ci du champ des libertés protégées ».

La protection du français au Québec ne justifie pas les atteintes à la liberté des commerçants de s'exprimer dans une langue de leur choix. Selon le Comité, «s'il est légitime qu'un Etat choisisse une ou plusieurs langues officielles, il ne l'est pas s'il supprime, en dehors de la vie publique, la liberté de s'exprimer dans une langue de son choix $^{191} »$.

La Charte n'a pas totalement rompu avec cette «conception classique des libertés ». Certaines de ses dispositions en rappellent la substance par le truchement des proscriptions d'interdire ${ }^{192}$ et d'obligation de faire ${ }^{193}$.

${ }^{189} \mathrm{CEDH}, 23$ juillet 1968 sur le régime linguistique de l'enseignement en Belgique, réponse à la première question, point $\mathrm{n}^{\circ} 7$, préc., supra note $\mathrm{n}^{\circ} 10$. ${ }^{190}$ Idem, pour la seconde question, point $\mathrm{n}^{\circ} 13$.

${ }^{191}$ Constatation du 31 mars 1993, § 11-3, n ${ }^{\circ} 5-6$, p. 961.

${ }^{192}$ Sur l'interdiction faite aux Etats de prohiber ou limiter l'emploi de langues infra-nationales dans des documents sur la vie économique et sociale, art. 13

PDLC n ${ }^{\circ}$ 13, 2002 
Dans le cadre de conflits inter-principes en France, l'encouragement d'une langue sub-nationale dans une zone d'influence ne saurait remettre en cause le principe d'égalité devant les services publics et devant la loi. Dans le domaine de l'enseignement, ce principe limite la liberté de choix de la langue. La valorisation des langues et cultures régionales est circonscrite aux seules disciplines spécifiques, le législateur ayant retenu la langue d'Etat comme celle de l'enseignement, des examens, des concours, de soutenance des thèses et des mémoires, sous réserve des nécessités de l'enseignement des langues et cultures régionales ${ }^{194}$. En opérant une différenciation selon les matières, la Charte va plus loin. Outre la discipline linguistique spécifique, l'enseignement dans des langues régionales ou minoritaires est encouragé en $^{195}$ car il suscite des adaptations tels les cours d'histoire et de culture dont ces parlers sont l'expression ${ }^{196}$ et la formation initiale des enseignants ${ }^{197}$.

En France, la tendance au cantonnement à une discipline linguistique spécifique s'inscrit dans la problématique centrale de l'admission d'un enseignement facultatif d'une langue et de la culture sub-étatiques. C'est une condition de la non-contrariété vis-à-vis du principe d'égalité des élèves scolarisés dans les établissements concernés eu égard aux droits et obligations applicables à tous les usagers des établissements assurant le service public de l'enseignement ou associés à celui-ci ${ }^{198}$. Le caractère facultatif de l'enseignement

$\S 1$ lettre a) de la Charte européenne des langues régionales ou minoritaires ; sur l'interdiction d'insérer des clauses en en excluant ou restreignant le recours dans les règlements internes des entreprises et les actes privés, tout au moins entre les locuteurs de la même langue, idem, art. $13 \S 1$ lettre b).

${ }^{193}$ Sur l'obligation de s'opposer aux pratiques tendant à décourager leur emploi dans le cadre des activités économiques ou sociales, idem, art. $13 \S 1$ lettre c).

${ }^{194}$ Art. 11 de la L. $\mathrm{n}^{\circ}$ 94-665 du 4 août 1994, préc., supra note $\mathrm{n}^{\circ} 41$.

${ }_{195}$ Art. 8 \& 1 lettre e), iii de la Charte européenne des langues régionales ou minoritaires.

${ }^{196}$ Idem, art. 8 § 1 lettre g).

${ }^{197}$ Idem, art. 8 \& 1 lettre h)

${ }^{198}$ Déc. 91-290 DC du 9 mai 1991, cons. n 37 ; JORF, 14 mai 1991, p. 6350.

\section{$\operatorname{PDLC~n}^{\circ}$ 13, 2002}


s'impose dans son principe (donc au législateur) et dans ses modalités de mise en œuvre (donc au pouvoir réglementaire national ou local ${ }^{199}$ ).

A priori, la liberté du mode d'expression commande le recours à ces langues pour l'exercice d'autres droits de l'homme (droits de citoyens et devoirs civiques ${ }^{200}$ ). Mais, cette position n'est pas évidente dans tous les cas. La Cour européenne des droits de l'homme a reconnu que des électeurs francophones d'une région linguistique néerlandaise n'étaient pas privés de leurs droits électoraux par le fait qu'ils devaient voter pour des candidats parlant néerlandais car ceux-ci devaient prêter serment au Parlement belge en néerlandais auquel cas ils ne siégeraient pas au Conseil flamand ${ }^{201}$. L'article $29 \S 1$ de la loi du 5 août 1980 autorisait les électeurs francophones de l'arrondissement de Hal-Vilvorde à porter leurs suffrages sur un candidat francophone pourvu qu'il parle le néerlandais ${ }^{202}$. La restriction quant à la liberté de choix s'accompagnait d'une autre en matière de représentativité. Les arguments soulevés par cette juridiction européenne sont pour la plupart captieux. Ni le caractère transitoire de la loi, ni le principe de territorialité, ni l'expérience d'autres Etats en la matière n'empêchent la transgression de la liberté d'expression de l'opinion du peuple sur le choix du corps électoral conformément à l'article 3 du Protocole $\mathrm{n}^{\circ} 1$. Une discrimination horizontale accompagnée d'une limitation du choix de la langue a été déterminante dans la fixation des conditions d'accès à une instance politique, d'où la stupéfaction devant la conclusion selon laquelle les électeurs français et néerlandais «jouissent des droits de vote et d'éligibilité dans les mêmes conditions légales ».

${ }^{99}$ Déc. $\mathrm{n}^{\circ}$ 2001-454 DC du 17 janvier 2002, cons. ${ }^{\circ} 25$

${ }^{200} \mathrm{http} / / /$ conventions.coe.int/treaty/fr/Reports/Html/148.htm, § 100, p. 14

201 CEDH, 2 mars 1987, Mathieu Mohin et Clerfayt c./Belgique, A 113,

Req. $\mathrm{n}^{\circ} 00009267 / 81$, préc., supra note $\mathrm{n}^{\circ} 10$

${ }^{202}$ Idem, points $\mathrm{n}^{\circ} 44$ et 56 .

PDLC n ${ }^{\circ}$ 13, 2002 
2. La recherche d'un statut juridique des langues régionales ou minoritaires

Face aux conflits «intra» et «inter-principes», les Etats n'étaient pas tenus de prendre des mesures positives d'incitation aux termes de l'article 10 de la $\mathrm{CEDH}^{203}$. L'assimilation de la non-entrave à une expression idiomatique plurale à un droit de l'homme était insatisfaisante. Ce constat est conforté par la liberté des Etats de déterminer le statut d'une langue, plus particulièrement de définir la ou les langue(s) officielle(s) dans les rapports avec les autorités publiques $^{204}$. La nécessité d'une convention spécifique garantissant des mesures positives explique l'existence de la Charte complétant les droits linguistiques compris dans la $\mathrm{CEDH}^{205}$ et celle de la Convention-cadre qui consacre un droit général à un usage libre d'une langue minoritaire «en privé comme en public, oralement ou par écrit ${ }^{206} »$.

Les mesures positives de la Charte répondent à la finalité de sauvegarde car, sans protection, les langues concernées sont amenées à disparaître $^{207}$. Dans cette logique de survie ${ }^{208}$, l'idée d'un statut des langues régionales ou minoritaires supplante l'application des simples principes de liberté et d'égalité. Cette orientation maximaliste vise à mettre en œuvre des droits personnels ou collectifs destinés à des groupes d'individus.

\footnotetext{
${ }^{203}$ Déc. du 26 juillet 1993, Annuaire IV, p. 332.

${ }^{204}$ Déc. du 13 juillet 1979, X. c./Irlande, Rec. 35, p. 137.

${ }^{205}$ Conseil de l'Europe, La Charte européenne des langues régionales ou minoritaires,

http://www.local.coe.int/inc.asp ? L=F\&M=\$t/212-5-0-5/minlang $/ \& M=\$ t / 212-$ 5-0-5/minla..., p. 1

Art. $10 \S 1$ de la Convention-cadre sur la protection des minorités nationales; art. $7 \& 1$ de la proposition de protocole additionnel à la Convention de sauvegarde des droits et libertés fondamentales concernant les personnes appartenant à des minorités nationales, http://stars.coe.fr/ta/ta93/frec1201.htm, p. 4

${ }^{07}$ Préambule et art. $7 \S 1$ lettre c) de la Charte européenne des langues régionales ou minoritaires.
}

${ }^{208} \mathrm{http}: / /$ conventions.coe.int/treaty/fr/Reports/Html/148.htm, § 63, p. 9.

\section{$\operatorname{PDLC~} n^{\circ} 13,2002$}


La Charte fait reposer la pérennisation d'un pluralisme linguistique sur l'imprescriptibilité d'un droit de pratiquer une langue dans la vie privée ou publique. La propension internationale à consacrer l'usage de langues régionales ou minoritaires comme un « droit » vise à améliorer leur statut juridique en dissociant les droits personnels inaliénables (droit de reconnaissance dans une communauté linguistique, etc.) des droits collectifs des groupes linguistiques (droit à l'enseignement, à disposer de services culturels, de moyens de communication $^{209}$, etc.). Les réalités sont parfois plus complexes. Le système onomastique intéresse les droits personnels quant aux anthroponymes et les droits collectifs quant aux toponymes et odonymes $^{210}$. L'adaptation du système d'anthroponyme impliquant l'emploi du patronyme et du prénom dans la langue minoritaire ${ }^{211}$ dérive du droit intuitu personae au nom. Le droit à leur reconnaissance officielle $^{212}$ découle d'un droit personnel. Sur la base de la loi de ratification de la Convention-cadre, l'Allemagne a adopté une loi sur le changement de nom des minorités pour que les intéressés puissent adapter leur ancien nom aux caractéristiques spécifiques de leur langue par le truchement d'une déclaration au greffier de l'état-civil ${ }^{213}$. Si le droit à reconnaissance officielle d'une langue minoritaire ${ }^{214}$ est un «droit collectif», le droit à reconnaissance dans une communauté linguistique reste attaché à la personne.

Dans le cadre de la CEDH, la Cour a statué sur une demande de changement de nom d'un ressortissant d'un Etat appartenant à une

${ }^{209}$ Art. 3 de la Déclaration universelle des droits linguistiques de juin 1996, in http://www.egt.ie/udhr/udlr-fr.html, p. 4.

210 Art. $11 \S 3$ de la Convention-cadre sur la protection des minorités nationales.

${ }^{211}$ Idem, art. $11 \S 1$.

${ }^{212}$ Art. $7 \S 2$ de la proposition de protocole additionnel à la Convention de sauvegarde des droits et libertés fondamentales concernant les personnes appartenant à des minorités nationales, préc., supra note $\mathrm{n}^{\circ} 14$

${ }^{213}$ Land de Basse-Saxe, préc., supra note $\mathrm{n}^{\circ} 104$, v. spéc. p. 4.

214 Art. $1 \S 1$ de la Convention-cadre sur la protection des minorités nationales.

$\operatorname{PDLC} n^{\circ}$ 13, 2002 
minorité linguistique ${ }^{215}$. Ce n'était pas la consonance suédoise du patronyme d'un finlandais qui était en cause, mais les conditions du bénéfice d'une modification de nom conforme à son euphonie originelle $^{216}$. Au motif qu'il n'y a aucune certitude que les ancêtres aient porté ledit nom de manière constante ${ }^{217}$, le requérant essuya un refus des autorités finnoises ${ }^{218}$. La volonté d'échapper à un harcèlement ne rend pas illicite ce refus qui ne viole pas le droit au respect de la vie privée ${ }^{219}$, ni ne discrimine sur le fondement de l'article $14^{220}$. En l'espèce, le lien affectif était ténu car le nom originel a été porté en dernier lieu par un ancêtre décédé deux siècles auparavant ${ }^{221}$.

L'incitation à recourir aux langues infra-étatiques dans la «vie publique » recouvre une double réalité, l'une sociétale faisant primer la liberté individuelle, l'autre administrative, encadrée par le droit interne. La Convention-cadre s'y intéresse mais davantage sur l'aspect des droits de la personne (droit, dans sa langue minoritaire, de présenter des enseignes, inscriptions et autres informations à caractère privé exposées à la vue du public ${ }^{222}$, de trouver des dénominations traditionnelles locales, noms des rues et autres indications topographiques destinés au public ${ }^{223}$, d'établir et d'entretenir des contacts transfrontaliers en cas d'accointances linguistiques similaires $^{224}$, etc.)

La promotion extrême des langues sub-étatiques passe par leur officialisation. Outre une «égalité verticale » circonscrite à la zone

${ }^{215}$ CEDH, 25 novembre 1994, Aff. Stjerna c./Finlande, Req. $n^{\circ}$ 0018131/91, A 299-B.

${ }^{216}$ Idem, point $\mathrm{n}^{\circ} 9$.

${ }^{217}$ Idem, point $\mathrm{n}^{\circ} 14$.

${ }^{218}$ Idem, point $\mathrm{n}^{\circ} 9$.

${ }^{219}$ Idem, point $\mathrm{n}^{\circ} 41$.

${ }^{220}$ Idem, point $\mathrm{n}^{\circ} 51$

${ }^{221}$ Idem, point $\mathrm{n}^{\circ} 43$.

${ }^{222}$ Art. 11 \& 2 de la Convention-cadre sur la protection des minorités nationales.

${ }^{223} \mathrm{Idem}$, art. $11 \S 3$

${ }^{224}$ Idem, art. $17 \S 1$

$\operatorname{PDLC~n}^{\circ}$ 13, 2002 
d'influence considérée, la qualité de « langue officielle » d'une langue infra-nationale confère des «droits subjectifs » liés à son emploi ${ }^{225}$. En Espagne, les garanties en matière d'usage normal et officiel des langues castillanes et régionales dépendent des mesures de la Communauté autonome créant les conditions d'une pleine égalité quant aux droits et devoirs linguistiques des citoyens de la Communauté concernée.

En Croatie, le régime juridique des langues minoritaires est lié à l'importance locale d'une communauté ou minorité linguistique. Le droit de leurs membres «d'utiliser librement leur langue et leur alphabet dans le contexte tant public que privé » peut s'accompagner, conformément à l'article $7,2^{\text {ème }}$ alinéa de la loi constitutionnelle sur les droits de l'homme et les droits des communautés ou minorités ethniques, d'un usage officiel de l'alphabet et de la langue communautaires ou minoritaires «dans les municipalités dans lesquelles (ils) [...] constituent la majorité de la population ${ }^{226} »$. A défaut de majorité, aucune langue minoritaire n'a reçu le statut de langue officielle régionale ${ }^{227}$. Par contre, l'article 8 de la loi constitutionnelle conférant aux autorités locales le soin de décider d'ériger une langue sub-étatique en langue officielle locale ${ }^{228}$ a été appliqué $^{229}$. Cette officialisation, limitée à une administration locale ${ }^{230}$ jugeant de l'opportunité d'y recourir ${ }^{231}$, ne s'impose pas aux instances régionales ${ }^{232}$

\footnotetext{
${ }^{225}$ http://cultura.gencat.es/llengcat/legis/LOH-1998-HTM, pp. 3-4

Application de la Charte européenne des langues régionales ou minoritaires

à la Croatie, 20 septembre 2001, ECRML (2001) 2, p. 10.

${ }^{227}$ Idem, § 21, p. 11

${ }^{228}$ Idem, § 22, p. 11

${ }^{229}$ Idem, § 79, p. 24

Idem, § 73, p. 22

${ }^{231}$ Idem, Concl., littera G, p. 32

${ }^{232}$ Idem, § 79, p. 24
}

$\operatorname{PDLC~n}^{\circ}$ 13, 2002 
$\mathrm{Au}$ final, parce que les principes du droit assurent à la fois la promotion et les restrictions à l'expression du pluralisme idiomatique intra-national, la disposition à préconiser un statut juridique dont la fonction est la seule promotion amène à les dépasser et à les moderniser. Mais, ces principes se retrouvent pleinement dans (et même confortés par) le statut juridique des langues infra-nationales. La co-officialisation, tout en garantissant la reconnaissance des « droits », constitue la mesure la plus proximale en terme d' "égalité verticale ». Le refus de nombreux pays de concevoir une co-officialisation ${ }^{233}$ égale à toutes les langues sub-étatiques et limitée géographiquement est la marque d'une aspiration à un encouragement maîtrisé de ces idiomes. L'invocation de motifs d'inconstitutionnalité à cet effet est empreinte du souci de préserver la suprématie de la langue d'Etat que seule altère une co-officialisation. Les positions étatiques oscillent entre conception classique et moderne des principes du droit et le droit international ne peut s'extirper de ces conflits d'intérêts. Or, éviter d'écourter la vie des langues infra-étatiques, n'est-ce pas l'une des missions essentielles du droit linguistique?

${ }^{233}$ Pour la France, le point de vue de vingt-cinq députés de droit in « Droit des minorités », Libération, 5 juillet 1999 ; déc. n 2030 du 28 novembre 1980 du Gouvernement polynésien proposant une co-officialisation du tahitien non entérinée par la France (Polynésie française,

http://www.ciral.ulaval.ca/alx/amlxmonde/pacifique/polfr.htm, p. 9).

$\operatorname{PDLC} n^{\circ}$ 13, 2002 